\title{
SYNTAX II and SYNTAX III trials: what is the take home message for surgeons?
}

\author{
Rodrigo Modolo ${ }^{1,2 \#}$, Carlos Collet ${ }^{1 \#}$, Yoshinobu Onuma ${ }^{3,4}$, Patrick W. Serruys ${ }^{5}$ \\ ${ }^{1}$ Department of Cardiology, Academic Medical Center, University of Amsterdam, Amsterdam, The Netherlands; ${ }^{2}$ Department of Internal Medicine, \\ Cardiology Division, University of Campinas (UNICAMP), Campinas, Brazil; ${ }^{3}$ Thoraxcenter, Erasmus University Medical Center, Rotterdam, The \\ Netherlands; ${ }^{4}$ Cardialysis Clinical Trials Management and Core Laboratories, Rotterdam, The Netherlands; ${ }^{5}$ International Centre for Circulatory \\ Health, NHLI, Imperial College London, London, UK \\ \#These authors contributed equally to this work. \\ Correspondence to: Prof. Patrick W. Serruys, MD, PhD. International Centre for Circulatory Health, NHLI, Imperial College London, London, UK. \\ Email: patrick.w.j.c.serruys@gmail.com.
}

\begin{abstract}
Percutaneous coronary intervention (PCI) has evolved greatly in the last 40 years since its introduction by Andreas Grüntzig in 1977. Since then, we've observed an evolution in balloons, the development of stents, changes in stent structure, development of drug eluting stents, improvements in strut design, thickness and even their polymeric coating. Most recently we saw the rise and "fall" of bioabsorbable scaffolds for PCI. Trials with the most diverse devices for PCI and diagnostic techniques have been conducted. Two of the most recent trials were reported in the last year and deserve special attention-SYNTAX II and SYNTAX III. These trials are completely different in design but present valuable information for doctors managing coronary artery disease (CAD). Both trials take into account contemporary technology for assessing and treating CAD. The first uses so-called "state-of-the-art" PCI and compares the outcomes of that approach with the outcomes of the PCI arm of the pivotal SYNTAX trial. SYNTAX III Revolution on the other hand does not focus on clinical endpoints: it is a blinded trial that does not randomize patients but randomizes doctors ("the heart team") to make a decision on the best treatment for complex CAD. This decision was based either on multi-slice CT with physiological assessment using $\mathrm{FFR}_{\mathrm{CT}}$ or on conventional angiography. In this review we bring the most important aspects of those trials and the key messages for surgeons together; also, what the surgeon may expect in the future after the publication of these interesting concepts.
\end{abstract}

Keywords: SYNTAX score; coronary artery disease (CAD); percutaneous coronary intervention (PCI); coronary artery bypass graft (CABG)

Submitted Jun 14, 2018. Accepted for publication Jul 11, 2018.

doi: 10.21037/acs.2018.07.02

View this article at: http://dx.doi.org/10.21037/acs.2018.07.02

\section{Introduction}

Over the past 40 years of percutaneous coronary intervention (PCI), the evolution in techniques and devices has been (and still is) tremendous. Still, the debate over the best approach for treating complex coronary artery disease (CAD) persists. In the last decade the SYNTAX trial showed superiority of surgery over percutaneous treatment using the firstgeneration drug-eluting stent $\left(\right.$ Taxus $^{\circledR}$ ) in three-vessel disease patients (3VD) $(1,2)$. For diabetic $3 \mathrm{VD}$ patients, this superiority was confirmed in the FREEDOM trial (3). With the use of new-generation drug-eluting stents, the BEST trial also demonstrated that PCI did not outrank CABG as the superior treatment for these subjects (4).

Regardless of the ongoing PCI vs. CABG comparisons, the field of percutaneous intervention has evolved, with development of new devices, technology and techniques. From balloon angioplasty, to implantation of stents, to the development of drug-eluting stents, to the redesign of shape and thickness of their struts, to bioabsorbable scaffolds, 
the field has continued to evolve since Andreas Grüntzig's work in 1977. This evolution has not been limited to device improvement, but has also involved adjunctive therapy, image guidance and physiological assessment of coronary stenosis.

The recent SYNTAX II trial (5) is a new milestone in the approach to $3 \mathrm{VD}$. This trial does not have the purpose of posing PCI against CABG, rather, it compares all the currently available techniques for the so-called "state-ofthe-art" PCI and analyses the improvement of PCI over time. This is done by comparing with the outdated PCI arm of the SYNTAX I trial (2).

Among diagnostic tools, the improvement in non-invasive imaging has enabled coronary computed tomography angiography (CCTA) to reach a high standard level of accuracy in assessing the anatomy of the diseased coronary artery (6) and its physiological significance via $\mathrm{FFR}_{\mathrm{CT}}(7)$. The hypothesis of the SYNTAX III Revolution trial is that a heart-team decision for the best CAD treatment (CABG or PCI) would be the same, whether assessing the coronary artery tree with new CCTA diagnostic tools or using conventional invasive angiography (8).

Although these two trials are completely different in concept and design, they gather information of utmost importance and interest for physicians who deal directly with CAD patients, especially cardiac surgeons performing coronary artery bypass graft (CABG). We aimed to review the main findings of these ground-breaking studies and to provide the surgical community with concise information stemming from these two trials.

\section{SYNTAX II trial}

\section{Design and patient selection}

The SYNTAX II trial is a multicenter, all-comers, openlabel, single-arm trial that screened patients with de novo $3 \mathrm{VD}$ who were candidates for revascularization (9) (Video 1).

It was designed to challenge the current guidelines' recommendation of only percutaneously treating patients with $3 \mathrm{VD}$ and an anatomical SYNTAX score of 22 or less. The moral justification to do so relies on the use of the SYNTAX score II. This score modulates the impact of the anatomical SYNTAX score in decision making according to the interaction of the clinical characteristics and comorbidities with purely anatomical equipoise (score of 15) that has been established after the SYNTAX I trial (9).

Recruitment occurred form February 2014 until November 2015 and included 454 patients. Patients were selected after screening from the local heart team (comprised of interventional cardiologists and cardiac surgeons) based on the recommendation of the SYNTAX score II, which had to demonstrate equipoise between PCI and CABG (i.e., both strategies were recommended based on the predicted 4-year mortality) (10). Patients were referred for PCI with a state-of-the-art approach, i.e., the revascularization was physiology guided, with the assistance of imaging guidance, use of novel stents and specialized personnel to treat CTO and bifurcation lesions.

\section{The SYNTAX score II}

The SYNTAX score II is a clinical tool that combines clinical variables with the anatomical SYNTAX score, providing expected 4-year mortality for both CABG and PCI-thus recommending either PCI only, CABG only or equipoise in treatment based on long-term mortality.

SYNTAX score II is based on interaction between clinical variables and the anatomical SYNTAX score using Cox model as the regression model, weighing the balance of PCI vs. CABG towards one or the other (Figure 1). The clinical variables included in the SYNTAX score II are age, renal function (creatinine clearance), left ventricle ejection fraction, left main involvement, sex, presence of chronic obstructive pulmonary disease and of peripheral vascular disease. The score is easily assessable via a web calculator (www.syntaxscore.com) (11) and follows the below nomogram for bedside application (Figure 2).

\section{State-of-the-art PCI}

Another interesting and innovative aspect of this trial is the so-called state-of-the-art approach for PCI. This approach combines physiologically guided intervention, the use of a novel thin strut $(70 \mu \mathrm{m}$, with abluminal biodegradable polymer coating stent-SYNERGY, Boston Scientific) and a mandatory post-PCI intravascular ultrasound (IVUS) assessment of adequate stent expansion and apposition (12).

Additionally, the treatment of bifurcation lesions was made according to the recommendation of the European Bifurcation Club's consensus (13) and chronic total occlusion (CTO) revascularization was preferably performed by a dedicated CTO operator.

\section{Physiology guided revascularization}

Physiology guided PCI consists of evaluating the functional 

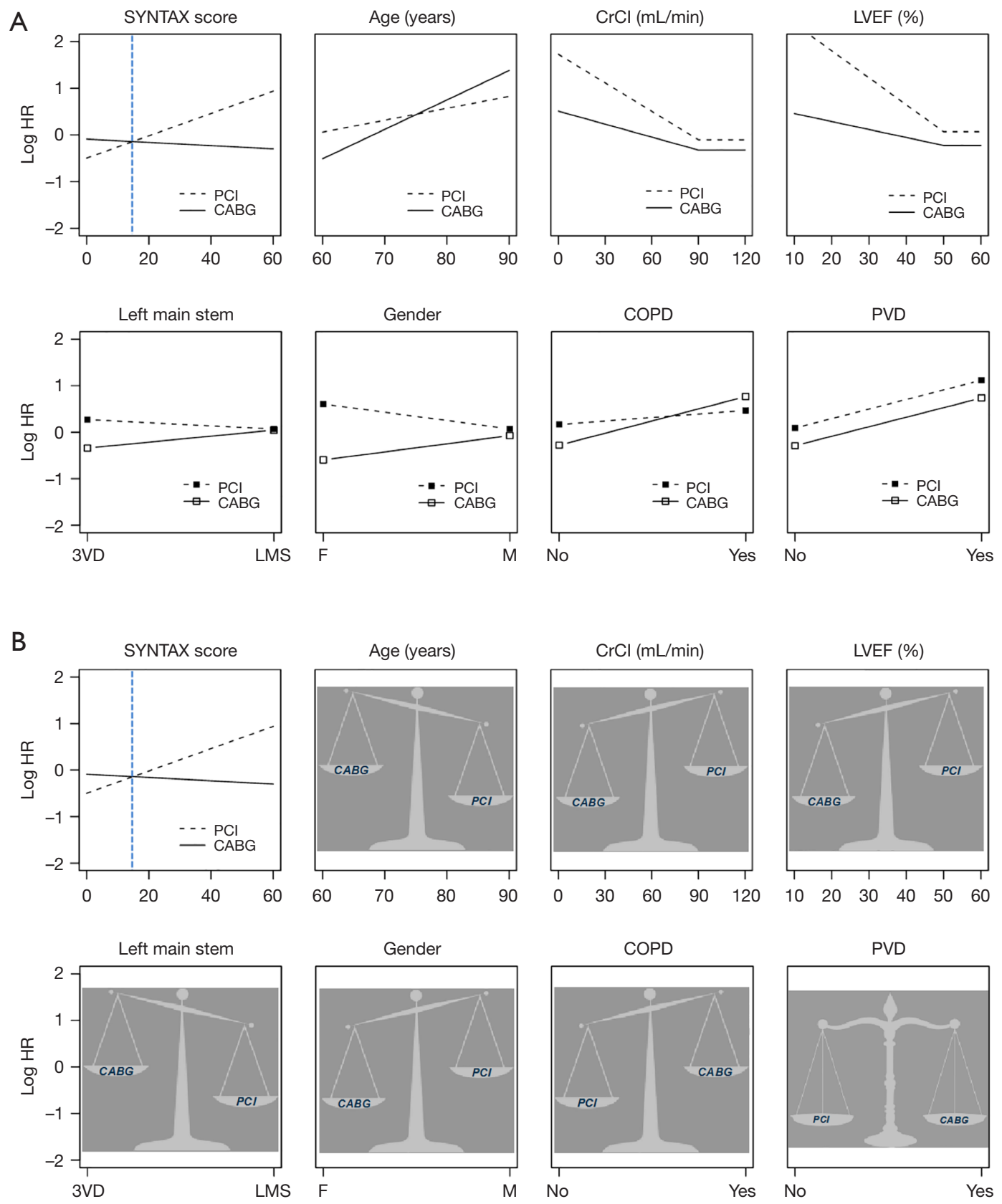

Figure 1 Demonstration of the concept of interaction-interaction between the mode of revascularization, anatomical SYNTAX score and the clinical characteristics and comorbidities. (A) Effects of the different variables are represented visually as a log hazard ratio for CABG (straight line) and PCI (dotted line) on the $y$-axis for each predictor. Each graph represents one predictor. Note the differing gradients of the hazards for PCI and CABG, leading to the hazards crossing at an anatomical SYNTAX score of 15 . At this cross-over point of hazards, the mortality risk is much the same between CABG and PCI. This threshold of cross-over of hazards will vary according to the variables-being lower for female sex, reduced LVEF, and younger age, and higher for COPD, left main disease, and older age. Peripheral vascular disease $(\mathrm{P}=1.00)$ equally affected mortality following each mode of revascularization (somewhat parallel lines). (B) the graphic representation using a weight scale balance-the weight represents the favorable effect (e.g., left main stem favors PCI). Reproduced and modified from Farooq et al. (10). CABG, coronary artery bypass surgery; PCI, percutaneous coronary intervention; HR, hazard ratio; CrCl, creatinine clearance; LVEF, left ventricular ejection fraction; left main, unprotected left main coronary artery disease; 3VD, three-vessel disease; LMS, left main stem; COPD, chronic obstructive pulmonary disease; PVD, peripheral vascular disease. 


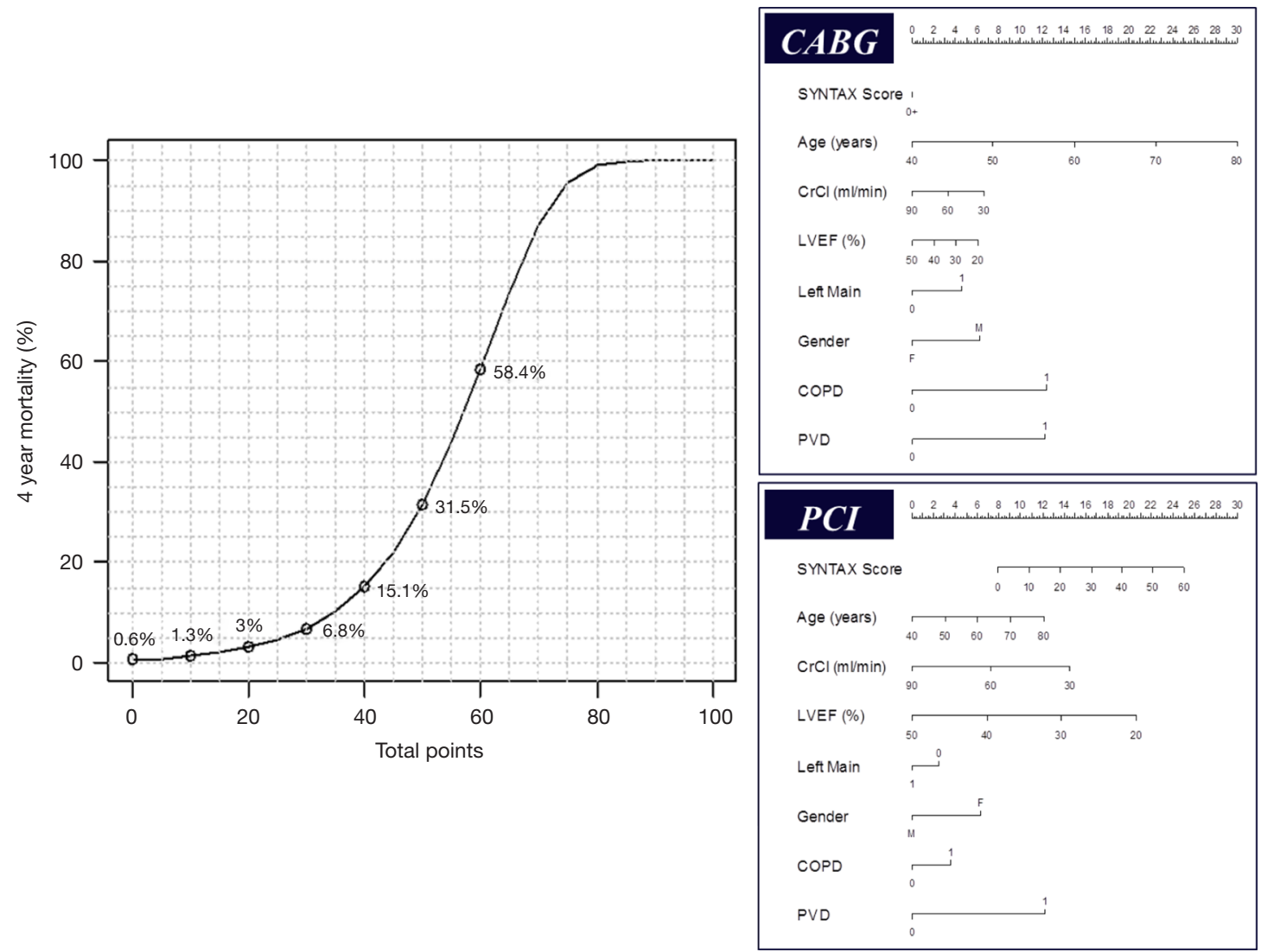

Figure 2 SYNTAX score II nomogram for offline application. With this nomogram the sum of points using the 8 factors (SYNTAX score, age, $\mathrm{CrCl}$, LVEF, left main, sex, COPD and PVD) can be used to assess the expected 4-year mortality for the individual patient either for CABG or PCI. For example, a 60-year-old man with an anatomical SYNTAX score of 30, unprotected left main coronary artery disease, creatinine clearance of $60 \mathrm{~mL} / \mathrm{min}$, an LVEF of 50\%, and COPD, would have 41 points (predicted 4-year mortality $16.3 \%$ ) to undergo CABG and 33 points (predicted 4-year mortality $8.7 \%$ ) to undergo PCI respectively. The same example without COPD included would lead to identical points (29 points) and 4-year mortality predictions (6.3\%) for CABG and PCI. Because of the rarity of complex coronary artery disease in premenopausal women, mortality predictions in younger women are predominantly based on the linear relation of age with mortality. The differences in mortality predictions in younger women between CABG and PCI will therefore be affected by larger 95\% CI than those in older women. Reproduced and modified from Farooq et al. (10). CABG, coronary artery bypass surgery; PCI, percutaneous coronary intervention; $\mathrm{CrCl}$, creatinine clearance; LVEF, left ventricular ejection fraction; left main, unprotected left main coronary artery disease; $3 \mathrm{VD}$, three-vessel disease; COPD, chronic obstructive pulmonary disease; PVD, peripheral vascular disease.

severity of a stenosis in the coronary artery to determine the need for revascularization. Currently it is performed using fractional flow reserve (FFR) or instantaneous wave-free ratio (iFR). FFR is assessed during maximal reactive hyperemia induced by intracoronary injection or intravenous infusion of adenosine (an off-label use of that compound). The administration of adenosine commonly causes the patient discomfort. The iFR is the instantaneous diastolic pressure gradient between the aorta and the pressure distal to the stenosis and does not necessitate the use of induced hyperemia. At the time of the design of the SYNTAX II trial, the equivalence of iFR and FFR was not yet established and was a topic of intense debate. Therefore, outside of a clear threshold for revascularization or deferral, there was a gray zone in measurement of iFR where it was recommended to perform an FFR with reactive hyperemia (Figure 3). The importance of this technology for functional assessment of a coronary lesion has been demonstrated in the large clinical trials FAME (14) and FAME 2 (15). In the FAME trial (including over one thousand patients) 


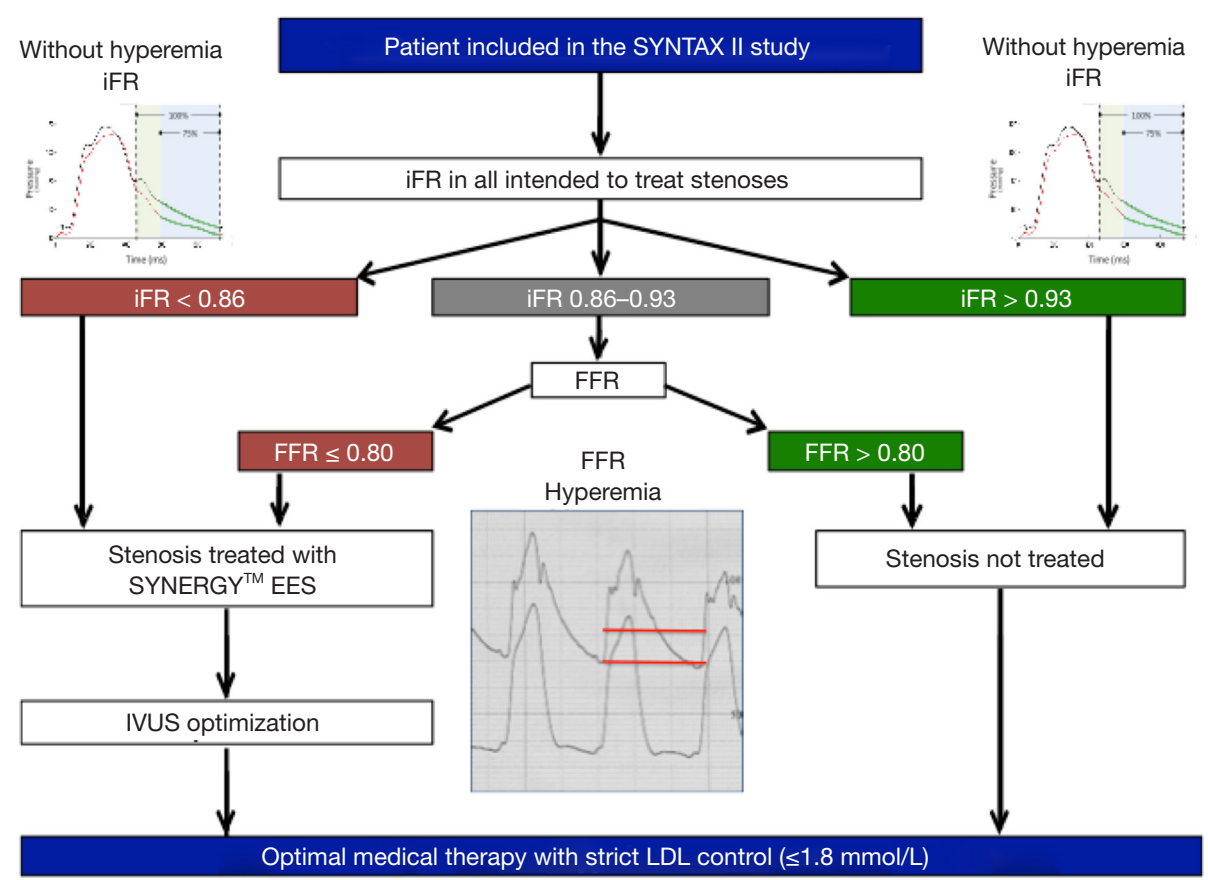

Figure 3 Flowchart of the SYNTAX II study for the physiological assessment. Instantaneous wave-free ratio (iFR) was performed in all lesions intended to be treated. If the assessment resulted in a ratio of less than 0.86 or more than 0.93 , no further action in regard to physiological assessment was necessary. For results within the grey zone (between 0.86 and 0.93 ), fractional flow reserve (FFR) assessment was required. iFR assesses the ratio between the pressures in the distal coronary and aorta $(\mathrm{Pd} / \mathrm{Pa})$ at a specific time frame called the wavefree period (in diastole), without the need for induced hyperemia (upper corners—right and left). FFR assesses the mean pressures (red lines) of the $\mathrm{Pd} / \mathrm{Pa}$ after the induced hyperemia with adenosine (center of the image).

the investigators demonstrated that compared with angiography-guided PCI, physiology-guided PCI (with FFR) was associated with lower rates of the composite endpoint of death, non-fatal myocardial infarction (MI), and repeat revascularization at 1 year, and also associated with fewer stents used per patient (physiology guided 1.9 1.3 stents $v s$. angiography guided $2.7 \pm 1.2$ stents, $\mathrm{P}<0.001$ ) in multivessel diseased patients.

A recent systematic review comprising 51,350 patients from 11 studies comparing physiology-guided versus angiography-guided PCI showed that the first is associated with lower likelihood of MI during hospitalization (OR $0.54,95 \%$ CI: $0.39-0.75, \mathrm{P}<0.001)$ and at follow-up (OR 0.53, 95\% CI: $0.40-0.70, \mathrm{P}<0.001)$, lower in-hospital (OR 0.51, 95\% CI: $0.37-0.70, \mathrm{P}<0.001$ ), and follow-up (OR 0.63, 95\% CI: $0.47-0.86, \mathrm{P}=0.004)$ major adverse cardiovascular events. Physiology-guided PCI also resulted in lower procedure cost (16).

Physiology guidance has proven to lower cardiovascular event rates not only for percutaneous revascularization but also for CABG. A recent 6-year follow-up study of 627 consecutive patients has shown that physiology-guided surgical revascularization is associated with a lower rate of all cause death or MI [16\% vs. $25 \%$; hazard ratio 0.59 (95\% CI: 0.38-0.93), $\mathrm{P}=0.020$ ] as compared with the conventional angiography-guided CABG (17). The iFR technique has the advantage of precluding the use of adenosine to achieve maximum hyperemia. iFR has been shown to be noninferior to FFR in two major clinical outcome trials-the DEFINE-FLAIR and the SWEEDHEART $(18,19)$.

\section{Main findings}

The SYNTAX II trial compared state-of-the-art PCI patients with a historical cohort of PCI patients from the SYNTAX I trial who had a matched "equipoise" predicted mortality at 4 years (5). Identical definitions of outcomes used in SYNTAX I were applied in the SYNTAX II trial. The primary outcome of the study was a composite endpoint of major adverse (patient-oriented) cardiac 


\begin{tabular}{|c|c|c|c|}
\hline Characteristics & SYNTAX II $(n=454)$ & SYNTAX I PCI arm $(n=315)$ & $P$ value \\
\hline Age (years) & $66.7 \pm 9.7[454]$ & $66.7 \pm 9.1[315]$ & 0.99 \\
\hline Male & $93.2 \%(423 / 454)$ & $93.0 \%(293 / 315)$ & 0.93 \\
\hline Body mass index $\left(\mathrm{kg} / \mathrm{m}^{2}\right)$ & $28.9 \pm 4.7[449]$ & $28.2 \pm 4.4[315]$ & 0.032 \\
\hline Diabetes mellitus type I or II & $30.3 \%(135 / 446)$ & $29.2 \%(92 / 315)$ & 0.75 \\
\hline Insulin treated & $8.5 \%(38 / 446)$ & $10.5 \%(33 / 315)$ & 0.36 \\
\hline Oral medication & $19.5 \%(87 / 446)$ & $16.8 \%(53 / 315)$ & 0.35 \\
\hline Diet only & $2.0 \%(9 / 446)$ & $1.9 \%(6 / 315)$ & 0.91 \\
\hline Current smoker & $14.7 \%(64 / 435)$ & $17.8 \%(56 / 315)$ & 0.26 \\
\hline Previous myocardial infarction & $12.5 \%(56 / 447)$ & $28.7 \%(89 / 310)$ & $<0.001$ \\
\hline Previous stroke & $5.6 \%(25 / 449)$ & $1.9 \%(6 / 315)$ & 0.01 \\
\hline Hypertension & $77.0 \%(344 / 447)$ & $73.4 \%(229 / 312)$ & 0.26 \\
\hline Hyperlipidemia & $77.3 \%(341 / 441)$ & $74.4 \%(232 / 312)$ & 0.35 \\
\hline Creatinine clearance (mL/min) & $82.0 \pm 26.9[454]$ & $87.3 \pm 28.5[315]$ & 0.008 \\
\hline Ejection fraction (\%) & $58.1 \pm 8.3[454]$ & $61.8 \pm 11.3[315]$ & $<0.001$ \\
\hline Peripheral vascular disease & $7.7 \%(35 / 454)$ & $9.5 \%(30 / 315)$ & 0.37 \\
\hline COPD & $10.8 \%(49 / 454)$ & $12.7 \%(40 / 315)$ & 0.42 \\
\hline Clinical presentation & & & $<0.001$ \\
\hline Silent ischemia & $6.7 \%(30 / 449)$ & $13.3 \%(42 / 315)$ & \\
\hline Stable angina & $68.8 \%(309 / 449)$ & $61.6 \%(194 / 315)$ & \\
\hline Unstable angina & $25.6 \%(115 / 449)$ & $25.1 \%(79 / 315)$ & \\
\hline Anatomic SYNTAX score & $20.3 \pm 6.4[454]$ & $22.8 \pm 8.7[315]$ & $<0.001$ \\
\hline SYNTAX score II PCI & $30.2 \pm 8.6[454]$ & $30.6 \pm 8.7[315]$ & 0.528 \\
\hline Predicted 4-year mortality PCI (\%) & $8.9 \pm 8.8[454]$ & $9.2 \pm 8.7[315]$ & 0.64 \\
\hline SYNTAX score II CABG & $29.1 \pm 10.4[454]$ & $29.1 \pm 9.6[315]$ & 1 \\
\hline Predicted 4-year mortality CABG (\%) & $9.0 \pm 9.3[454]$ & $8.5 \pm 8.1[315]$ & 0.44 \\
\hline
\end{tabular}

$\mathrm{PCl}$, percutaneous coronary intervention; CABG, coronary artery bypass graft; COPD, chronic obstructive pulmonary disease (Escaned et al. SYNTAX II).

and cerebrovascular events (MACCE or PoCE-patient oriented composite endpoint) at 1-year follow-up. Major adverse cardiac and cerebrovascular event was defined as allcause death, stroke, any MI or any revascularization. Also, there was an exploratory short-term and 5-year follow-up comparison of the SYNTAX II patients with the equipoisederived patients in the CABG arm of the SYNTAX I trial. An independent clinical event committee adjudicated all events.
The baseline characteristics of the patients in both groups are shown in Table 1. Although the anatomical SYNTAX score is different between the groups, the SYNTAX score II (4-year predicted mortality) was similar between PCI groups. Patients undergoing state-of-the-art PCI experienced less PoCE (10.6\% vs. 17.4\%; HR 0.58, 95\% CI: $0.39-0.85, \mathrm{P}=0.006)$ compared with the equipoise PCI arm of SYNTAX I. The difference was mainly driven by a significant reduction in the incidence of MI (HR 0.27 , 
95\% CI: 0.11-0.70, $\mathrm{P}=0.007$ ) and revascularization (HR 0.5 7, 95\% CI: $0.37-0.90, \mathrm{P}=0.015)$. Rates of all-cause death (HR 0.69, 95\% CI: $0.27-1.73, \mathrm{P}=0.43$ ) and stroke (HR 0.69, 95\% CI: $0.10-4.89, \mathrm{P}=0.71$ ) were similar (Figure 4). Another finding worthy of mentioning was the rate of stent thrombosis, which was lower with the SYNTAX II approach (HR 0.26, 95\% CI: 0.07-0.97, P=0.045). The results were confirmed by sensitivity analyses of the primary endpoint adjusted by confounding factors using the inverse probability of treatment weighting (IPTW) approach and multivariate Cox regression analysis. The confounding factors used on the IPTW approach were: gender, age, diabetes status, hyperlipidemia, previous MI, left ventricular ejection fraction, creatinine clearance, number and length of the stents implanted and anatomical SYNTAX score.

The exploratory analysis comparing SYNTAX II PCI patients with the equipoise SYNTAX I CABG arm (simulating a state-of-the-art PCI vs. CABG for multivessel disease patients) showed no difference between the groups with regards to PoCE $[10.6 \%$ vs. $11.2 \%$; HR 0.91 (95\% CI: 0.59-1.14), $\mathrm{P}=0.684$, respectively]. However, this comparison is merely exploratory; it compares contemporary state-of-the-art PCI patients with the historical (outdated) CABG cohort of the SYNTAX I trial. Thus, improvements in CABG techniques are not taken into account. Data not yet published comparing CABG patients from the EXCEL trial (20) with those from the SYNTAX I trial shows that the surgery has improved since SYNTAX.

Most recently, the eagerly awaited results of the 2-year follow-up of the SYNTAX II trial were presented at the EuroPCR 2018 Congress in Paris-these results are yet to be published. At this mid-term follow-up, the main results held, i.e., the SYNTAX II strategy was associated with improved clinical outcomes at two years, compared with the matched patients treated percutaneously in the original SYNTAX I trial. Also, the 2-year exploratory comparison between SYNTAX II and matched CABG patients from the "historical" SYNTAX I trial suggests equivalent outcomes for PCI with CABG when the SYNTAX II strategy is followed (Figure 5).

\section{Implication of the SYNTAX II trial for surgeons}

In the field of coronary revascularization, multiple studies compare CABG with PCI in order to find the best option for patients with CAD. SYNTAX II is not such a trial. However, it brings some important key messages for interventional cardiologists and also for the surgical community.

An important message is that we are making progress with the "holistic" approach for treating CAD with PCIhowever, we cannot attribute improvement in outcomes to any specific change in our percutaneous approach. The introduction of physiology-guided therapy suggests that revascularization based on physiology has a cardioprotective effect that could become relevant for surgeons performing revascularization [suggested by Fournier et al. (17) in CABG patients]. This is especially important in a medical world where new potent pharmacological agents targeting coronary artery plaque regression are constantly being introduced and improved.

The importance of the SYNTAX score II as a tool for heart team decision making must be mentioned, since this score represents an integrated approach in selecting patients for surgery or PCI.

\section{SYNTAX III Revolution trial}

\section{Heart team assessment}

Currently, the treatment of choice for patients with complex multivessel CAD is CABG (2-4,21). A heart team approach (i.e., a team comprised of cardiac surgeon, interventional cardiologist and clinical cardiologist) is recommended by international guidelines for deciding the between CABG and PCI for 3 VD patients $(1,22)$. This decision is based mainly on the complexity of the CAD assessed by the anatomical SYNTAX score compounded with the patient's comorbidities and clinical characteristics (23).

Until now, conventional coronary angiography was used for assessing CAD and for decision-making by the heart team. However, CCTA has arisen as a non-invasive tool capable of providing precise information on coronary stenosis and plaques (24-26). Furthermore, the physiological assessment of FFR non-invasively, with the use of the $\mathrm{FFR}_{\mathrm{CT}}$ has already been demonstrated to be accurate in patients with multivessel disease (27). Those were the basic concepts for the design of the SYNTAX III Revolution trial (Video 2).

\section{The trial}

The SYNTAX III Revolution trial tested the hypothesis that the heart team's treatment recommendation derived from coronary CTA is in agreement with the recommendation derived from conventional angiography 
A Major Adverse Cardiac or Cerebrovascular Events

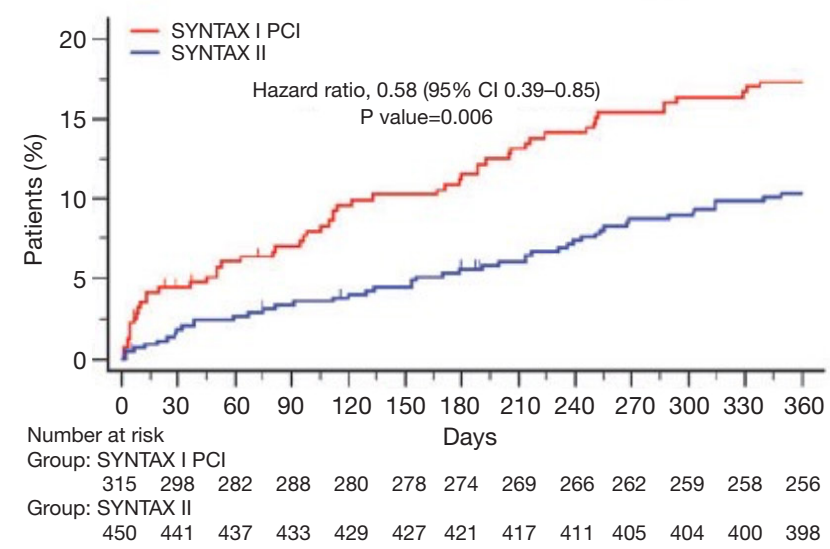

C

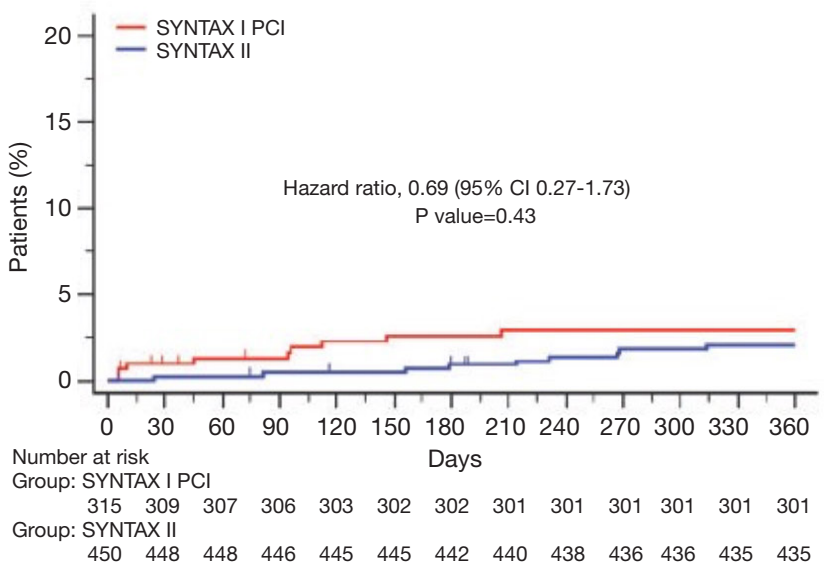

E

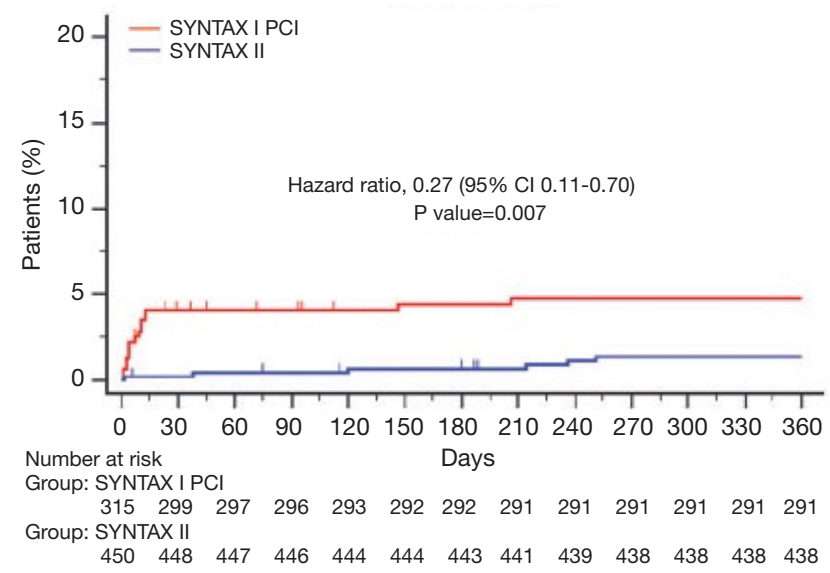

B

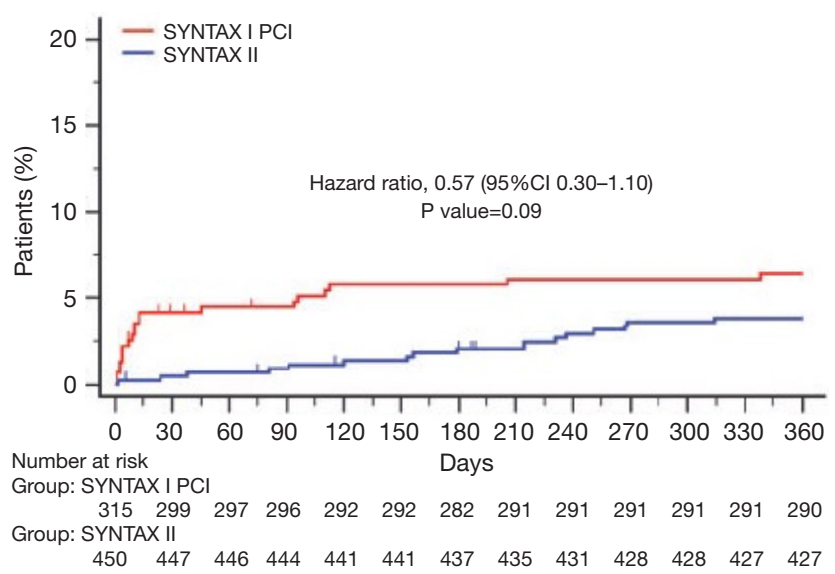

D

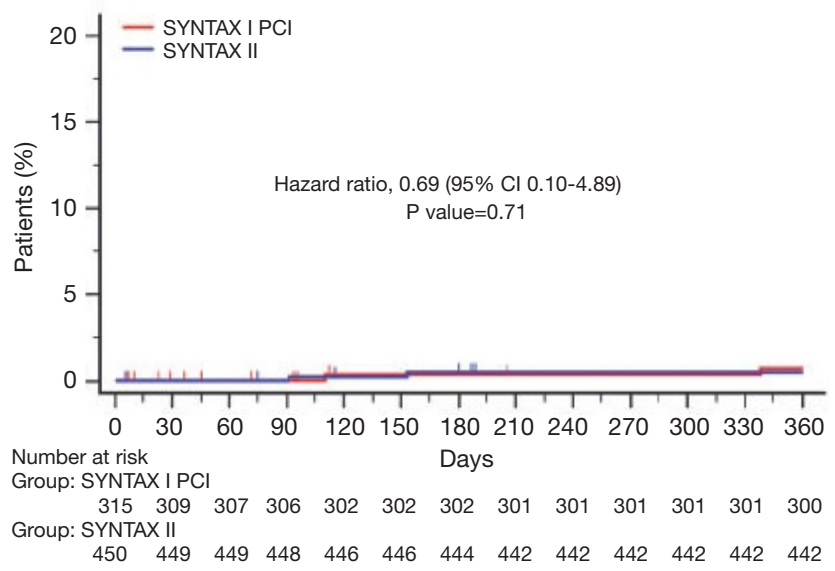

$\mathrm{F}$

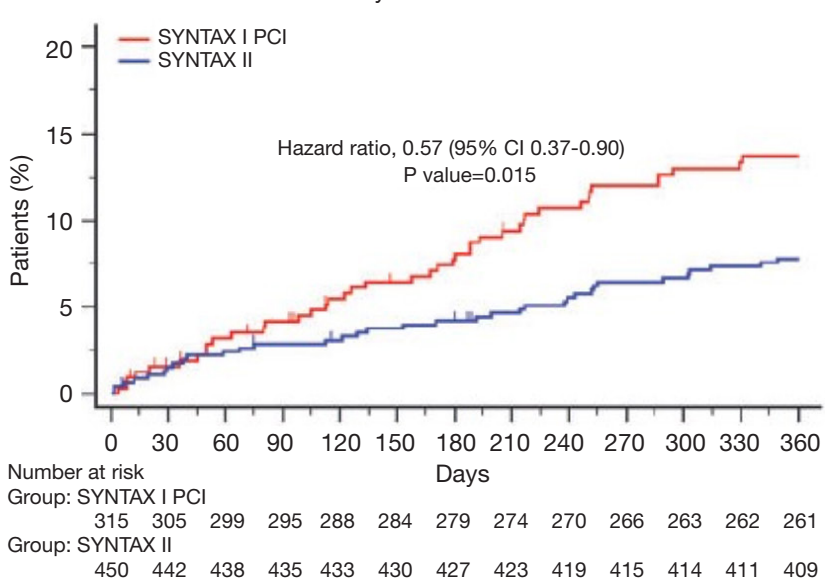

Figure 4 One-year clinical outcomes among the study patients, compared with the equipoise-derived SYNTAX I PCI cohort. The KaplanMeier curves show the event rate are for the SYNTAX II patients (blue) and the PCI arm of the SYNTAX I trial (red) regarding the composite primary (A), all-cause death/stroke/MI (B), all cause death (C); stroke (D); any myocardial infarction (E); any revascularization (F). Reproduced from Escaned et al. (5). 


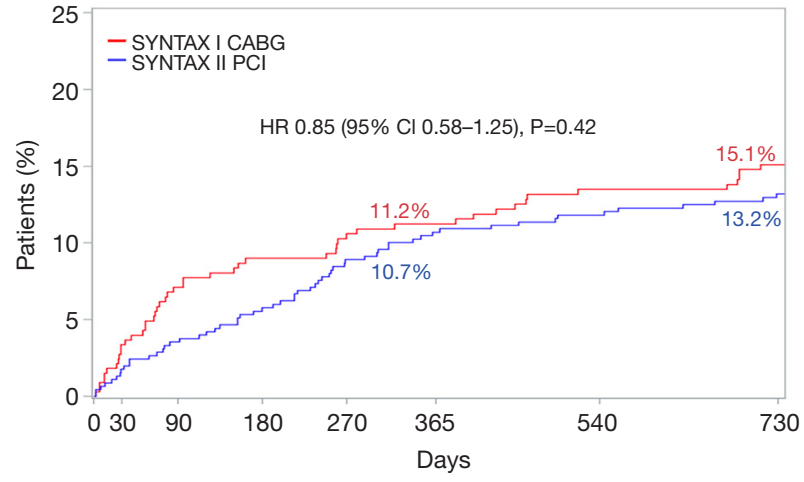

Number at risk SYNTAX I CABG $\begin{array}{llllll}334313 & 295 & 289 & 279 & 277 & 268\end{array}$ SYNTAX II PCI

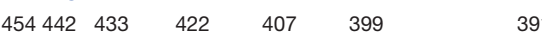
391

Figure 5 Two-year clinical outcomes among the study patients, compared with the equipoise-derived SYNTAX I CABG cohortexploratory analysis. Kaplan-Meier curves are shown for the SYNTAX II group (blue) and the surgical revascularization arm of the original SYNTAX I trial (red) for the major adverse cardiac or cerebrovascular events (MACCE). Presentation of SYNTAX II 2-year results at EuroPCR 2018, Paris, France.

in patients with left main or three-vessel CAD. To allow for the investigation of the agreement between two imaging diagnostic modalities on treatment recommendation (i.e., CABG or PCI), two heart teams were randomized to assess epicardial CAD either with coronary CTA or conventional coronary angiography in addition to clinical information. The heart teams comprised a cardiac surgeon, an interventional cardiologist and a radiologist. The primary end-point was agreement between two heart teams on treatment recommendation, i.e., agreement on one out of the following two options: (I) CABG only or (II) PCI only or equipoise between CABG and PCI, based on anatomical assessment of CAD. The heart team's treatment recommendation relied on 4-year mortality prediction using the SYNTAX score II (10).

A secondary end-point including the physiological component was introduced using FFR derived from coronary CTA (FFRCT). The SYNTAX score II, was re-calculated with the functional SYNTAX score and heart teams made a second treatment recommendation accounting for the flow limiting aspect of epicardial stenosis. Anatomical score was corrected for physiological significance and score points attributed to anatomical lesions are deducted whenever the stenotic lesion was not functionally significant (Anatomical score MINUS points for lesion not functionally significant EQUALS Functional SYNTAX score). The combination of the functional SYNTAX score with clinical characteristics and comorbidities is designated as the SYNTAX score III (Figure 6).

The SYNTAX III Revolution trial showed that coronary CTA evaluation with $\mathrm{FFR}_{\mathrm{CT}}$ was feasible in patients with multivessel disease in 196 out of 223 patients (87.9\%). Although the correlation between the anatomical SYNTAX score derived from cineangiography and multi-slice CT scan was moderate (Pearson coefficient of $0.59, \mathrm{P}<0.001-$ systematic error of 3.6), the SYNTAX score II derived from these imaging modalities had a high degree of correlation (Pearson coefficient of $0.99, \mathrm{P}<0.001$ - systematic error of 0.49 ). The reason for that correlation increase when calculating the SYNTAX score II may lie in the fact that, for CABG, the 'weight' of the anatomical SYNTAX score is null, thus not impacting the surgical risk.

Agreement between heart teams for recommended treatment using either CTA or conventional angiography was "almost perfect" Moreover, the agreement between Heart Teams on the epicardial coronary vessel to be revascularized reached $81 \%$.

The inclusion of $\mathrm{FFR}_{\mathrm{CT}}$ changed the treatment decision in $7 \%$ of the cases and modified treatment planning in $16 \%$ of the cases. The results of the SYNTAX III Revolution trial showed that treatment decision making between CABG and PCI based on coronary CTA is in in almost perfect agreement with the decision derived from conventional coronary angiography in patients with left main or $3 \mathrm{VD}$, supporting the potential role of non-invasive imaging with coronary CTA for treatment decision making and planning (Figure 7).

\section{Image acquisition}

CCTA imaging was obtained using the new generation CT scanner, 'Revolution CT' (GE Healthcare, Milwaukee, USA). The GE Revolution scanner has $160 \mathrm{~mm}$ coverage in the $\mathrm{z}$-axis and 0.28 -second rotation speed, allowing for acquisition of the whole heart within a single beat (28). Motion artefacts, if present, were corrected by postprocessing algorithm (28). Acquisitions were performed after administration of nitrates. Beta-blockers were recommended for heart rates $>65$ beats $/ \mathrm{min}$. The $\mathrm{FFR}_{\mathrm{CT}}$ analyses were provided by HeartFlow Inc. (Redwood City, CA, USA). 


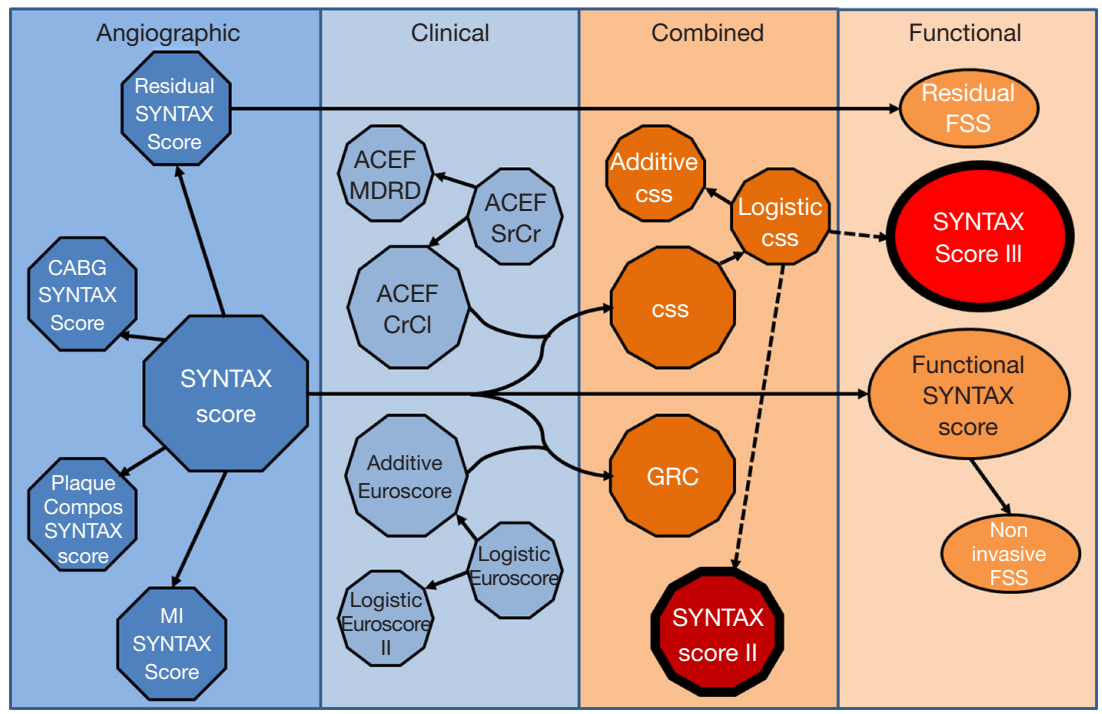

Figure 6 Evolution of the risk score algorithms derived from the historical SYNTAX I trial. ACEF, age, creatinine, ejection fraction; CABG, coronary artery bypass grafting; Compos, compositional; CrCl, creatinine clearance; CSS, Clinical SYNTAX score; FSS, Functional SYNTAX score; GRC, Global Risk Classification; MI, myocardial infarction; MDRD, Modification of Diet in Renal Disease; SrCr, serum creatinine; SYNTAX, Synergy between PCI with Taxus and Cardiac Surgery.

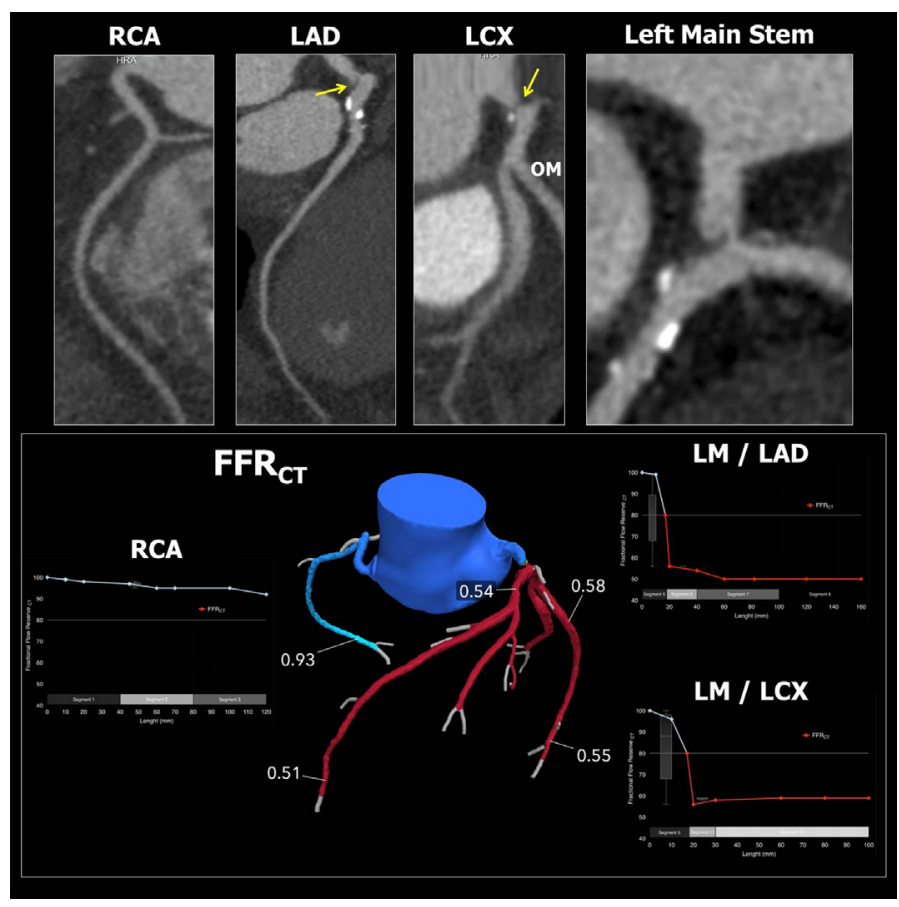

Figure 7 Case example of assessment of CAD using the coronary computed tomography with $\mathrm{FFR}_{\mathrm{CT}}$ for functional assessment. In this case there is no lesion in the right coronary artery, left descending coronary artery nor circumflex. However, a severe lesion can be seen in the distal left main stem (yellow arrows). In the lower panel a representation of FFRCT assessment is shown. From the left main stem to both the LAD and LCX there is a drop in FFR (reaching 0.51 and 0.55 , respectively). For this patient, the functional assessment agreed with the anatomical assessment. The Heart Teams decision were for CABG, based on the non-invasive assessment. CAD, coronary artery disease; CABG, coronary artery bypass grafting; LAD, left anterior descending; LCX, left circumflex; LM, left main; RCA, right coronary artery. 


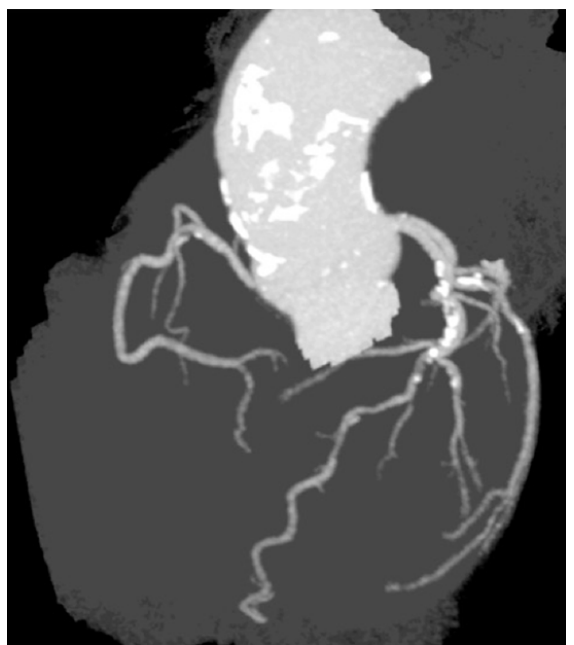

Figure 8 3D-maximum intensity projection (MIP). Coronary computed tomography CT reconstruction of the aortic root and coronary arteries showing intense calcification in the left descending coronary artery and also in the aortic root. This image warns the surgeon for adapting coronary artery bypass surgical technique due to the intense calcification of the aorta.

With this technology, a nominal spatial resolution of $230 \mu \mathrm{m}$ could be achieved providing information on the severity and extent of CAD comparable to conventional angiography (28). Moreover, emerging technology such as $\mathrm{FFR}_{\mathrm{CT}}$ added physiological information in all major coronary vessels (29). Epicardial coronary stenoses with lesion-specific FFR $_{\mathrm{CT}}$ $\leq 0.80$ were considered hemodynamically significant.

Coronary CTA were processed using three-dimensional maximal intensity projection images (MIP), multi-planar reconstructions and axial images. The anatomical SYNTAX score and SYNTAX score II were calculated in an independent core laboratory (Cardialysis BV, Rotterdam, The Netherlands) $(10,23)$.

\section{Feasibility of planning CABG relying only on coronary CTA}

For the surgical community, the feasibility to perform CABG based solely on coronary CTA and FFR $_{\text {CT }}$ would depend primarily on the precise recognition of a landing zone for the anastomosis of the graft in the coronary artery. Therefore, the non-invasive assessment of the entire coronary artery is crucial for planning CABG. The distal segment is important for assessing landing zones, while evaluation of the proximal artery segments does not impact on anatomical planning but plays a major role in the functional assessment of stenosis. Also, the surgeon's preoperative assessment of the feasibility of grafting the artery depends mainly on the quality and size of the target vessels, i.e., diameter and calcification pattern may be useful for surgical planning.

The most challenging scenario for coronary CTA is the presence of calcified plaques that (due to the blooming artefact) hamper luminal evaluation (30). Calcified stenosis located in the proximal segment of the coronary arteries does not pose a problem for surgeons because the anastomoses are performed at the distal segments of the coronary arteries. Furthermore, in several cases included in the trial, the presence of calcification at the level the aortic root warned the surgical team that manipulation at this level or cross-clamping might be associated with a higher risk of stroke, leading them to adapt a surgical technique aimed at reducing periprocedural complications (31-33) (Figure 8).

\section{Implication of the SYNTAX III Revolution study for the surgical community}

In patients with multivessel disease, the guidelines recommend a multidisciplinary heart team discussion, taking into consideration the anatomical complexity of the CAD, as well as the patient's clinical characteristics and comorbidities $(1,22)$. For decades, the gatekeeper for surgical revascularization has been the cardiologist performing diagnostic coronary angiography. In patients with low anatomical complexity, assessed by an anatomical SYNTAX score $\leq 22$, PCI can be considered as an alternative to CABG $(1,22)$. In patients with anatomical SYNTAX score $>22$, CABG provides superior clinical outcomes compared to PCI (2). SYNTAX III Revolution incorporates the radiologist into the heart team discussion, potentially shifting the gatekeeping process to the radiologist and allowing for treatment decisions and planning based solely on non-invasive imaging. This may potentially impact surgical practice, integrating non-invasive imaging and heart team's discussion before the patient goes to the catheterization laboratory or the operative room.

Surgical revascularization planning is customarily based on the anatomical severity of coronary stenosis. Recently, several trials have demonstrated the benefit of guiding revascularization based on the physiological repercussions of anatomical stenosis. FFR-guided CABG is associated with a significant reduction in the rate of overall death or MI 
at 6-year follow-up as compared with angiography-guided CABG (17). Nonetheless, systematic three-vessel pressure wire evaluation to determine FFR or other resting index is time consuming and seldom performed in clinical practice. The assessment of $\mathrm{FFR}_{\mathrm{CT}}$ before surgical revascularization has the potential to increase physiology-guided CABG, potentially improving clinical outcomes after surgical revascularization.

\section{Conclusions}

The SYNTAX III Revolution trial was a decisionmaking study and the decision of the heart teams were not bound to the revascularization strategy performed. It was demonstrated that treatment decisions were the same, regardless of whether coronary CTA or coronary angiography were used for evaluation by the heart team, however, whether this same equipoise would occur between imaging modalities in the real-world setting remains to be confirmed. A clinical outcome randomized trial comparing a coronary CTA with $\mathrm{FFR}_{\mathrm{CT}}$ strategy versus standard of care is required in order to introduce this strategy in routine clinical practice.

\section{Acknowledgements}

Studies reported within this article received grants from Boston Scientific, Volcano, Philips, GE Healthcare and HeartFlow.

\section{Footnote}

Conflicts of Interest: C Collet received grants from HeartFlow and Biosensors. PW Serruys is consultant for Philips. R Modolo and Y Onuma have no conflicts of interest to declare.

\section{References}

1. Authors/Task Force members, Windecker S, Kolh P, et al. 2014 ESC/EACTS Guidelines on myocardial revascularization: The Task Force on Myocardial Revascularization of the European Society of Cardiology (ESC) and the European Association for CardioThoracic Surgery (EACTS)Developed with the special contribution of the European Association of Percutaneous Cardiovascular Interventions (EAPCI). Eur Heart J 2014;35:2541-619.
2. Serruys PW, Morice MC, Kappetein AP, et al.

Percutaneous coronary intervention versus coronary-artery bypass grafting for severe coronary artery disease. $\mathrm{N}$ Engl J Med 2009;360:961-72.

3. Farkouh ME, Domanski M, Sleeper LA, et al. Strategies for multivessel revascularization in patients with diabetes. N Engl J Med 2012;367:2375-84.

4. Park SJ, Ahn JM, Kim YH, et al. Trial of everolimuseluting stents or bypass surgery for coronary disease. $\mathrm{N}$ Engl J Med 2015;372:1204-12.

5. Escaned J, Collet C, Ryan N, et al. Clinical outcomes of state-of-the-art percutaneous coronary revascularization in patients with de novo three vessel disease: 1-year results of the SYNTAX II study. Eur Heart J 2017;38:3124-34.

6. Miller JM, Rochitte CE, Dewey M, et al. Diagnostic performance of coronary angiography by 64-row CT. N Engl J Med 2008;359:2324-36.

7. Douglas PS, Pontone G, Hlatky MA, et al. Clinical outcomes of fractional flow reserve by computed tomographic angiography-guided diagnostic strategies vs. usual care in patients with suspected coronary artery disease: the prospective longitudinal trial of $\mathrm{FFR}(\mathrm{CT})$ : outcome and resource impacts study. Eur Heart J 2015;36:3359-67.

8. Cavalcante R, Onuma Y, Sotomi $Y$, et al. Non-invasive Heart Team assessment of multivessel coronary disease with coronary computed tomography angiography based on SYNTAX score II treatment recommendations: design and rationale of the randomised SYNTAX III Revolution trial. EuroIntervention 2017;12:2001-8.

9. Campos CM, Stanetic BM, Farooq V, et al. Risk stratification in 3-vessel coronary artery disease: Applying the SYNTAX Score II in the Heart Team Discussion of the SYNTAX II trial. Catheter Cardiovasc Interv 2015;86:E229-38.

10. Farooq V, van Klaveren D, Steyerberg EW, et al. Anatomical and clinical characteristics to guide decision making between coronary artery bypass surgery and percutaneous coronary intervention for individual patients: development and validation of SYNTAX score II. Lancet 2013;381:639-50.

11. Sotomi Y, Collet C, Cavalcante R, et al. Tools and Techniques - Clinical: SYNTAX score II calculator. EuroIntervention 2016;12:120-3.

12. de Jaegere P, Mudra H, Figulla H, et al. Intravascular ultrasound-guided optimized stent deployment. Immediate and 6 months clinical and angiographic results from the Multicenter Ultrasound Stenting in Coronaries Study (MUSIC Study). Eur Heart J 1998;19:1214-23.

13. Lassen JF, Holm NR, Banning A, et al. Percutaneous coronary intervention for coronary bifurcation disease: 
11 th consensus document from the European Bifurcation Club. EuroIntervention 2016;12:38-46.

14. Tonino PA, De Bruyne B, Pijls NH, et al. Fractional flow reserve versus angiography for guiding percutaneous coronary intervention. N Engl J Med 2009;360:213-24.

15. De Bruyne B, Pijls NH, Kalesan B, et al. Fractional flow reserve-guided PCI versus medical therapy in stable coronary disease. N Engl J Med 2012;367:991-1001.

16. Enezate T, Omran J, Al-Dadah AS, et al. Fractional flow reserve versus angiography guided percutaneous coronary intervention: An updated systematic review. Catheter Cardiovasc Interv 2017. doi: 10.1002/ccd.27302.

17. Fournier S, Toth GG, De Bruyne B, et al. Six-Year Follow-Up of Fractional Flow Reserve-Guided Versus Angiography-Guided Coronary Artery Bypass Graft Surgery. Circ Cardiovasc Interv 2018;11:e006368.

18. Davies JE, Sen S, Dehbi HM, et al. Use of the Instantaneous Wave-free Ratio or Fractional Flow Reserve in PCI. N Engl J Med 2017;376:1824-34.

19. Götberg M, Christiansen EH, Gudmundsdottir IJ, et al. Instantaneous Wave-free Ratio versus Fractional Flow Reserve to Guide PCI. N Engl J Med 2017;376:1813-23.

20. Stone GW, Sabik JF, Serruys PW, et al. EverolimusEluting Stents or Bypass Surgery for Left Main Coronary Artery Disease. N Engl J Med 2016;375:2223-35.

21. Yusuf S, Zucker D, Peduzzi P, et al. Effect of coronary artery bypass graft surgery on survival: overview of 10year results from randomised trials by the Coronary Artery Bypass Graft Surgery Trialists Collaboration. Lancet 1994;344:563-70.

22. Patel MR, Calhoon JH, Dehmer GJ, et al. ACC/AATS/ AHA/ASE/ASNC/SCAI/SCCT/STS 2017 Appropriate Use Criteria for Coronary Revascularization in Patients With Stable Ischemic Heart Disease: A Report of the American College of Cardiology Appropriate Use Criteria Task Force, American Association for Thoracic Surgery, American Heart Association, American Society of Echocardiography, American Society of Nuclear Cardiology, Society for Cardiovascular Angiography and Interventions, Society of Cardiovascular Computed Tomography, and Society of Thoracic Surgeons. J Am Coll Cardiol 2017;69:2212-41.

23. Sianos G, Morel MA, Kappetein AP, et al. The SYNTAX Score: an angiographic tool grading the complexity of coronary artery disease. EuroIntervention 2005;1:219-27.

24. Collet C, Onuma Y, Grundeken MJ, et al. In vitro validation of coronary CT angiography for the evaluation of complex lesions. EuroIntervention 2018;13:e1823-30.
25. Douglas PS, Hoffmann U, Patel MR, et al. Outcomes of anatomical versus functional testing for coronary artery disease. N Engl J Med 2015;372:1291-300.

26. Onuma Y, Collet C, van Geuns RJ, et al. Long-term serial non-invasive multislice computed tomography angiography with functional evaluation after coronary implantation of a bioresorbable everolimus-eluting scaffold: the ABSORB cohort B MSCT substudy. Eur Heart J Cardiovasc Imaging 2017;18:870-9.

27. Collet C, Miyazaki Y, Ryan N, et al. Fractional Flow Reserve Derived From Computed Tomographic Angiography in Patients With Multivessel CAD. J Am Coll Cardiol 2018;71:2756-69.

28. Andreini D, Pontone G, Mushtaq S, et al. Image quality and radiation dose of coronary CT angiography performed with whole-heart coverage CT scanner with intracycle motion correction algorithm in patients with atrial fibrillation. Eur Radiol 2018;28:1383-92.

29. Nørgaard BL, Leipsic J, Gaur S, et al. Diagnostic performance of noninvasive fractional flow reserve derived from coronary computed tomography angiography in suspected coronary artery disease: the NXT trial (Analysis of Coronary Blood Flow Using CT Angiography: Next Steps). J Am Coll Cardiol 2014;63:1145-55.

30. Vavere AL, Arbab-Zadeh A, Rochitte CE, et al. Coronary artery stenoses: accuracy of 64-detector row CT angiography in segments with mild, moderate, or severe calcification--a subanalysis of the CORE-64 trial. Radiology 2011;261:100-8.

31. Bar-El Y, Goor DA. Clamping of the atherosclerotic ascending aorta during coronary artery bypass operations. Its cost in strokes. J Thorac Cardiovasc Surg 1992;104:469-74.

32. Mickleborough LL, Walker PM, Takagi Y, et al. Risk factors for stroke in patients undergoing coronary artery bypass grafting. J Thorac Cardiovasc Surg 1996;112:12508; discussion 1258-9.

33. Roach GW, Kanchuger M, Mangano CM, et al. Adverse cerebral outcomes after coronary bypass surgery.

Multicenter Study of Perioperative Ischemia Research Group and the Ischemia Research and Education Foundation Investigators. N Engl J Med 1996;335:1857-63.

Cite this article as: Modolo R, Collet C, Onuma Y, Serruys PW. SYNTAX II and SYNTAX III trials: what is the take home message for surgeons? Ann Cardiothorac Surg 2018;7(4):470482. doi: $10.21037 /$ acs.2018.07.02 